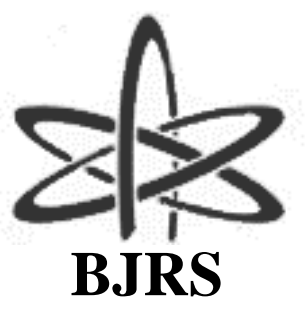

BRAZILIAN JOURNAL

$\mathrm{OF}$

RADIATION SCIENCES

07-02A (2019) 01-12

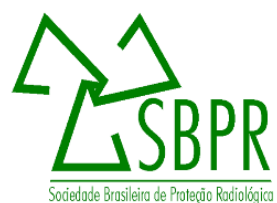

\title{
EVALUATION OF DIGITAL DETECTOR ARRAYS SYSTEMS FOR INDUSTRIAL RADIOGRAPHY
}

\author{
Aline S. S. Silva ${ }^{1}$, Davi F. Oliveira ${ }^{1,2}$, Célio S. Gomes ${ }^{1}$, Soraia R. Azeredo ${ }^{1}$ and Ricardo \\ T. Lopes ${ }^{1}$
}

${ }^{1}$ Laboratório de Instrumentação Nuclear/COPPE

Universidade Federal do Rio de Janeiro

68509 Rio de Janeiro, RJ

aline@lin.ufrj.br

davi@lin.ufrj.br.br

celio@lin.ufrj.br

soraia@lin.ufrj.br

ricardo@lin.ufrj.br

${ }^{2}$ Departamento de Física Aplicada - Instituto de Física

Universidade do Estado do Rio de Janeiro

Rua São Francisco Xavier, 524

20550-900 Rio de Janeiro, RJ

davi.oliveira@uerj.br

\begin{abstract}
Digital Detector Arrays (DDA) or Flat Panel Detector (FPD) is a highly efficient technique that is used in nondestructive testing of internal features of an object. The evaluation of DDA systems for industrial radiography is important to ensure the image quality and to enables long-term stability of this system. This evaluation is specified by ASTM E2737-10, which describes the fundamental parameters of DDA systems to be measured. The tests require the usage of either the five-groove wedge or the duplex plate phantom with separate Image Quality Indicators (IQIs). The purpose of this work was evaluate the radiographic performance achieved using both techniques in two DDA systems manufactured by GEIT: DXR250P and DXR250V, which have thallium-doped cesium iodide (CsI:Tl) and terbium-doped gadolinium oxysulfide $\left(\mathrm{Gd}_{2} \mathrm{O}_{2} \mathrm{~S}: \mathrm{Tb}-\mathrm{GOS}\right)$ scintillators, respectively. For this purpose, it was used an X-ray equipment as radiation source. The image quality parameters analyzed were Image Lag (IL), Offset Level (OL), Bad Pixel distribution, Burn In (BI), Spatial Resolution (SR), Material Thickness Range (MTR), Contrast Sensitivity (CS), Signal Level (SL) and Signal-to-Noise Ratio (SNR). As result of this study, has been observed that the use of the five-groove wedge phantom made the measurements to become easier to execute. Regarding the DDA system, the DXR250P presented more IL and BI, but produced images with better CS and SNR and needed a dose almost twice smaller than the DXR250V to achieve a similar SL.
\end{abstract}

ISSN: 2319-0612

Accept Submission: 2018-10-29 


\section{INTRODUCTION}

Digital radiography is one of the most used nondestructive testing (NDT) techniques for a number of industrial applications. Digital Detector Arrays (DDA) or Flat Panel Detectors (FPD) offer a straight digitization of the radiographic image. This technology presents high quality images with many possibilities of post-processing filters. DDA may operate in direct or indirect way of converting incident radiation into electrical charge that can be read out. Direct detectors convert the absorbed $\mathrm{x}$-rays into charge directly in a photoconductor. Indirect detectors first convert $\mathrm{x}$-rays to visible light in a scintillator and, then, detect the visible light in a photosensor array. Each method has advantages and disadvantages, as well as special limits of use in imaging systems [1-4].

Indirect detectors use a photosensor built into each pixel and the entire array is covered by a scintillating layer, where X-ray interacts and produces visible light. These light photons are detected by a matrix of photodiodes fixed on an amorphous silicon ( $\mathrm{a}-\mathrm{Si}$ ) layer and the electric charges generated within every photodiode are read by an active matrix of thin-filmtransistor (TFT) [5,6]. In the industrial area, the most used scintillators are terbium-doped gadolinium oxysulfide $\left(\mathrm{Gd}_{2} \mathrm{O}_{2} \mathrm{~S}: \mathrm{Tb}\right.$ - GOS) and thallium-doped cesium iodide (CsI:Tl). The combination of these scintillators with the a-Si photodiode has one of the largest Detection Quantum Efficiency (DQE) [7].

The quality of a digital image is affected by several factors including exposure and detector parameters and imaging software. According to the level of accuracy and sensitivity desired to the DDA performance, a routine evaluation should be carried out. Due to the possible degradation over time of the digital radiographic system, it is important to control its performance to ensure the maintenance of the quality and sensitivity of the generated images.

The aim of this work was evaluate the radiographic performance achieved in two DDA systems, with different scintillators, according to the standard ASTM E2737-10 [8], which establishes the fundamental parameters to be measured to ensure the image quality using: the duplex plate phantom with separate Image Quality Indicators (IQIs) or the five-groove wedge phantom. For this purpose, the following measurements were performed: Image Lag (IL), Offset Level (OL), Bad Pixel distribution, Burn In (BI), Spatial Resolution (SR), Material Thickness Range (MTR), Contrast Sensitivity (CS), Signal Level (SL) and Signal-to-Noise Ratio (SNR).

\section{MATERIALS AND METHODS}

The evaluation of DDA systems was performed using two different methodologies. The first one uses the duplex plate phantom, that consists in two steel plates of $6 \mathrm{~mm}$ and $9 \mathrm{~mm}$, so that the thickest part of the phantom is $15 \mathrm{~mm}$. This technique requires the use of duplex-wire and hole type IQIs $[9,10]$ (Figure 1a). The second methodology uses the five-groove wedge phantom [8], that consists in a continuous steel wedge, with five long grooves on one side and thicknesses ranging from 3 to $25 \mathrm{~mm}$ (Figure 1b). 


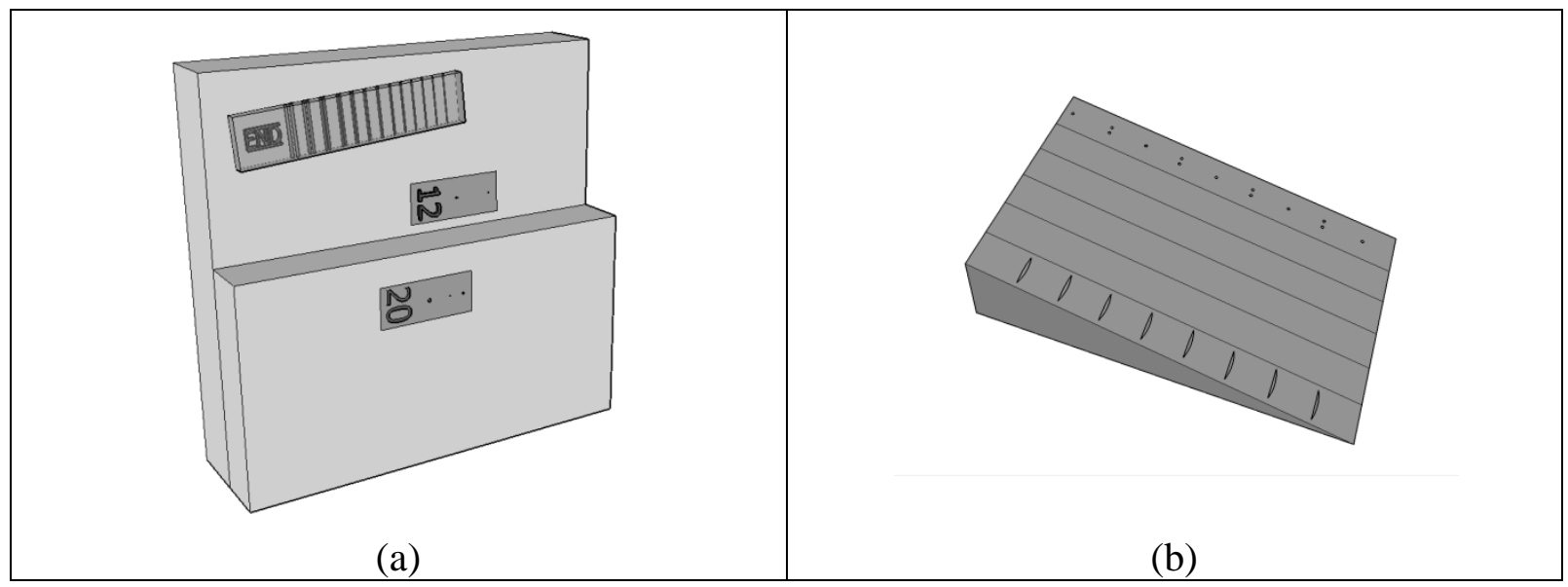

Figure 1: Design of the (a) duplex plate phantom with separate IQIs and (b) five-groove wedge phantom.

The DDAs systems analyzed were DXR250P and DXR250V, which have CsI:Tl and GOS scintillators, respectively, both manufactured by GEIT, with a bit depth of 14 bits for signal codification and $200 \mu \mathrm{m}$ pixel size. Before starting the performance testing, each DDA was calibrated for offset and gain to generate corrected images per manufacturer's recommendation, in exception of the IL and OL tests. Besides that, the images collected for the tests were corrected for bad pixels, in accordance with Standard ASTM E2597 [11].

Figure 2 shows the experimental setup used in this study. The measurements have been made using an X-ray tube model MGC41 (Yxlon), with focal spot size of $1.0 \mathrm{~mm}$ and maximum high voltage of $160 \mathrm{kV}$. The phantoms were placed directly on the FPD, which was positioned at $600 \mathrm{~mm}$ from the X-ray source. Table 1 shows the exposure parameters for both DDA systems.

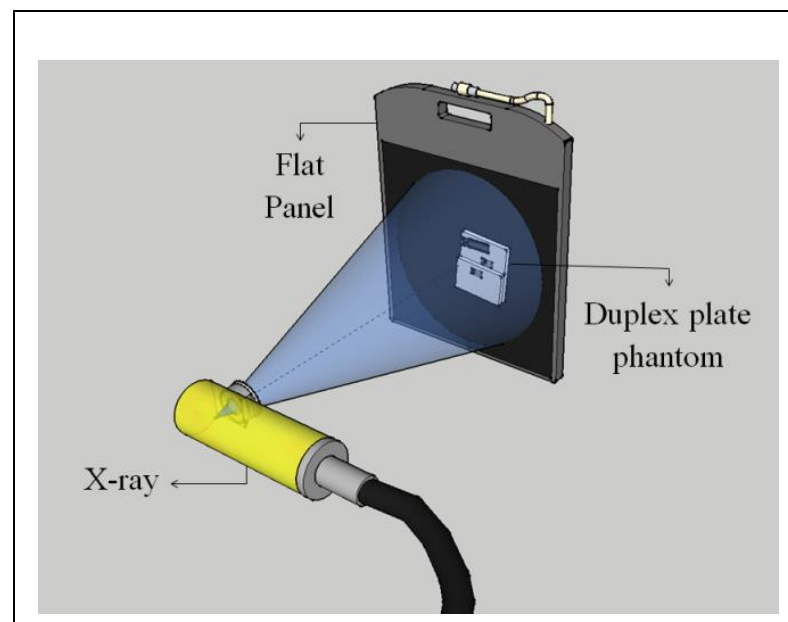

(a)

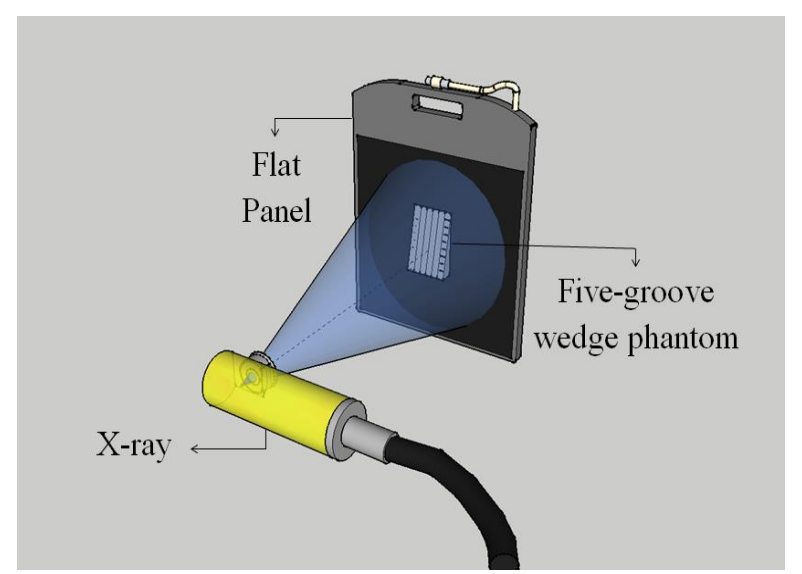

(b)

Figure 2: Experimental setup using (a) the duplex plate phantom with separate IQIs and (b) the five-groove wedge phantom.

Table 1: Exposure parameters for DXR250P and DXR250V. 


\begin{tabular}{c|c|c|c|c|c}
\hline \multirow{2}{*}{ DDA } & Tests & $\begin{array}{c}\text { High } \\
\text { Voltage }(\mathbf{k V})\end{array}$ & $\begin{array}{c}\text { Current } \\
(\mathbf{m A})\end{array}$ & $\begin{array}{c}\text { Integration } \\
\text { Time }(\mathbf{s})\end{array}$ & Dose (mGy) \\
\hline \multirow{4}{*}{ DXR250P } & IL & 130 & 0.1 & 10 & 0.73 \\
\cline { 2 - 6 } & BI & 130 & $0.1 / 0.01$ & 10 & $0.73 / 0.053$ \\
\cline { 2 - 6 } & $\begin{array}{c}\text { Other tests with } \\
\text { phantom }\end{array}$ & 130 & 3 & 10 & 19.96 \\
\cline { 2 - 6 } & OL & - & - & 30 & - \\
\cline { 2 - 6 } & IL & 130 & 0.4 & 10 & 2.69 \\
\cline { 2 - 6 } & BI & 130 & $0.4 / 0.04$ & 10 & $2.69 / 0.22$ \\
\hline & $\begin{array}{c}\text { Other tests with } \\
\text { phantom }\end{array}$ & 130 & 7 & 10 & 46.32 \\
\cline { 2 - 6 } & OL & - & - & 30 & - \\
\hline
\end{tabular}

The digital radiographic images were evaluated through the software ISee (version 10.2) [12] and the following measurements were performed in accordance with the standard ASTM E2737-10 [8]: Image Lag (IL), Offset Level (OL), Bad Pixel distribution, Burn In (BI), Spatial Resolution (SR), Material Thickness Range (MTR), Contrast Sensitivity (CS), Signal Level (SL) and Signal-to-Noise Ratio (SNR). The description of these measurements is below and the tests involving phantoms are described in items 2.1 and 2.2. The BI test, despite using phantom, has the same methodologies for both techniques.

- Image Lag (IL): The DDA shall be exposed with a constant dose rate with the selected energy at about $80 \%$ of saturation gray value $(\mathrm{GV})$. The lag of the detector was measured using three images: an offset image (L0), captured without radiation, an image (L1) captured with $10 \mathrm{~s}$ of total integration time and an image (L2) captured while shutting down the Xrays. A Region of Interest (ROI) of about 50 by 10 pixels was drawn at the same position in all images and the mean GV was noted. IL was calculated as the quotient of both GV corrected by the offset value, according to eq. (1).

$$
I L \%=\frac{G V(L 2)-G V(L 0)}{G V(L 1)-G V(L 0)} \times 100
$$

- Offset Level (OL): One image with about $30 \mathrm{~s}$ of integration time was captured without radiation and a ROI of about $90 \%$ of the active area of the DDA was drawn. OL is the mean signal level in the ROI.

- Bad Pixel distribution: All relevant clusters were noted and Bad Pixel Map stored.

- Burn In (BI): For this measurement, first the DDA containing phantom was exposed for ten minutes with $80 \%$ of saturation GV of the DDA in the area without the phantom. After that, the phantom was removed and the DDA was exposed at the same energy but at a tenth of the original exposure dose. An image with $10 \mathrm{~s}$ total exposure time was captured (BI1). The measurement was repeated after ten minutes without further exposure between measurements (BI10). Two ROIs were drawn, one in the thicker region of the phantom and another in the region outside the phantom in BI1 and BI10 images. The BI was calculated by the difference in signal of both regions divided by the signal in the region outside the phantom. 


\subsection{The evaluation of DDA systems using the duplex plate phantom with separate IQIs}

- Spatial Resolution (SR): The SR was determined using a duplex-wire IQI, placed directly on the phantom, in the region of smaller thickness. The largest wire pair with less than $20 \%$ dip resolution provides the SRmin.

- Material Thickness Range (MTR): This test is done from the calculation of the Contrast to Noise Ratio (CNR), through the hole IQI in the thicker region of the phantom. The CNR was calculated by the difference in signal due to the $2 \mathrm{~T}$ hole divided by the noise around the hole. The MTR is determined by the condition of CNR > GBV, where GBV is the required contrast of the hole IQI.

- Contrast Sensitivity (CS): For this test was used two hole type IQIs, each one placed directly on the surface of the phantom, in both regions (smaller and larger thickness). The CS was calculated according to eq. (2), where $\mathrm{MT}_{\text {Total }}$ is the thickness of the step plus the IQI and $\mathrm{MT}_{\mathrm{IQI}}$ is the thickness of the IQI.

$$
C S \%=\frac{G B V}{C N R} x \frac{M T_{I Q I}}{M T_{\text {Total }}} \times 100
$$

- Signal level (SL): The SL was determined by selecting four regions of interest (ROIs) of size 50 x 50 pixels, two in the thinner area (ROI 1 and ROI 2) and two in the thicker area (ROI 3 and ROI 4) of the phantom. The mean signal values of the each area were calculated.

- Signal to Noise Ratio (SNR): The SNR was measured through the same four regions of interest mentioned above (ROIs 1 - 4), by dividing the SL by the median standard deviation (noise).

\subsection{The evaluation of DDA systems using the five-groove wedge phantom}

- Spatial Resolution (SR): The $\mathrm{SR}_{\min }$ is the smallest long groove that is visible in the image at the smallest material thickness.

- Material Thickness Range (MTR): The MTR is calculated by drawing a line profile with 11 pixels width perpendicular to the groove, beginning at the smallest thickness and moving the line profile to the thicker material area until the smallest groove visible disappears into the noise.

- Contrast Sensitivity (CS): The CS was calculated by dividing the diameter of the selected groove by the MTR.

- Signal Level (SL): The SL is calculated by drawing two ROIs, one in the thinnest area and another in the MTR area of the five-groove wedge. The SL is the median single line gray value.

- Signal to Noise Ratio (SNR): The SNR is the quotient of the SL by the noise, for each ROI described above. 


\section{RESULTS}

From the experimental measurements performed, it was possible to obtain the values of the quality parameters for each DDA system analyzed. Table 2 shows the results obtained of IL, OL, BI, SR, MTR, CS, SL and SNR for DXR250P and DXR250V systems.

Comparing the results achieved from the duplex plate phantom and the five-groove wedge phantom, it is possible to observe that the BI and CS values were similar for both techniques. The differences of MTR, SL and SNR values are explained by the dimensions of each phantom. The duplex plate phantom has the highest thickness of $15 \mathrm{~mm}$, so the maximum MTR is $15 \mathrm{~mm}$. Through the five-groove wedge phantom it was possible to observe that the DDAs systems analyzed can reach an MTR of $20 \mathrm{~mm}$.

Table 2: Image quality parameters achieved to DXR250P and DXR250V

\begin{tabular}{|c|c|c|c|c|c|}
\hline \multirow{2}{*}{\multicolumn{2}{|c|}{ Image Quality Parameters }} & \multicolumn{2}{|c|}{ DXR250P } & \multicolumn{2}{|c|}{ DXR250V } \\
\hline & & $\begin{array}{c}\text { Duplex Plate } \\
\text { Phantom }\end{array}$ & $\begin{array}{c}\text { Five-groove } \\
\text { Wedge }\end{array}$ & $\begin{array}{c}\text { Duplex Plate } \\
\text { Phantom }\end{array}$ & $\begin{array}{c}\text { Five-groove } \\
\text { Wedge }\end{array}$ \\
\hline \multicolumn{2}{|c|}{$\operatorname{IL}(\%)$} & \multicolumn{2}{|c|}{4.0} & \multicolumn{2}{|c|}{1.3} \\
\hline \multicolumn{2}{|c|}{$\mathrm{OL}$} & \multicolumn{2}{|c|}{1,972} & \multicolumn{2}{|c|}{2,022} \\
\hline \multirow{2}{*}{$\mathrm{BI}(\%)$} & BI1 & 1.2 & 1.3 & 0.82 & 0.94 \\
\hline & BI10 & 0.91 & 1.0 & 0.66 & 0.67 \\
\hline \multicolumn{2}{|c|}{$\mathrm{SR}(\mu \mathrm{m})$} & 160 & 127 & 160 & 127 \\
\hline \multicolumn{2}{|c|}{$\operatorname{MTR}(\mathrm{mm})$} & 15 & 20 & 15 & 20 \\
\hline \multirow{2}{*}{ CS (\%) } & Thin & 0.752 & \multirow{2}{*}{0.635} & 0.825 & \multirow{2}{*}{0.705} \\
\hline & Thick & 0.686 & & 0.769 & \\
\hline \multirow{2}{*}{ SL } & Thin & 10,870 & 12,202 & 9,239 & 10,800 \\
\hline & Thick & 2,548 & 1,102 & 1,043 & 389 \\
\hline \multirow{2}{*}{ SNR } & Thin & 185 & 498 & 107 & 477 \\
\hline & Thick & 65 & 178 & 56 & 123 \\
\hline
\end{tabular}

The SR values had greater difference between the techniques for evaluation of the system, since they were obtained from different procedures. The duplex plate phantom use the duplex-wire IQI and the five-groove wedge phantom use their grooves for estimating this 
parameter. Figure 3 shows the procedures performed for the evaluation of quality parameters using both phantoms.

The differences in values of the quality parameters achieved for each technique does not mean that one is better or more precise than the other, but rather that they use distinct methodologies to calculate the same parameter. The duplex plate phantom uses IQIs for the estimation of these values, while the five-groove wedge phantom enables to obtain them directly, without the use of IQIs and mathematical calculations.

The standard [8] allows both techniques to be used for the performance evaluation of a DDA system, however, in order to make a comparison of the system behavior and, consequently, a verification of its long-term stability, is important to maintain the selected technique.

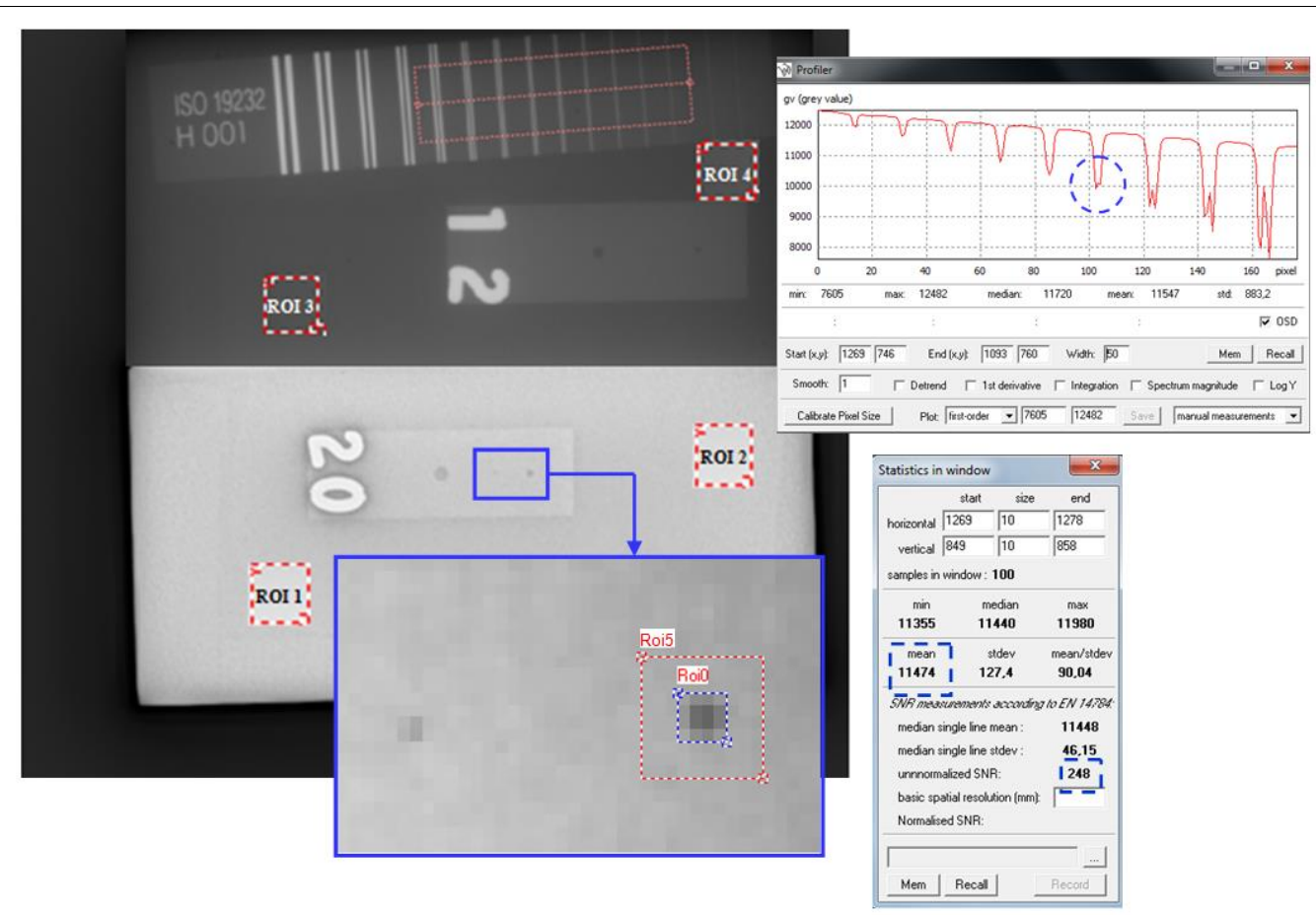

(a) 


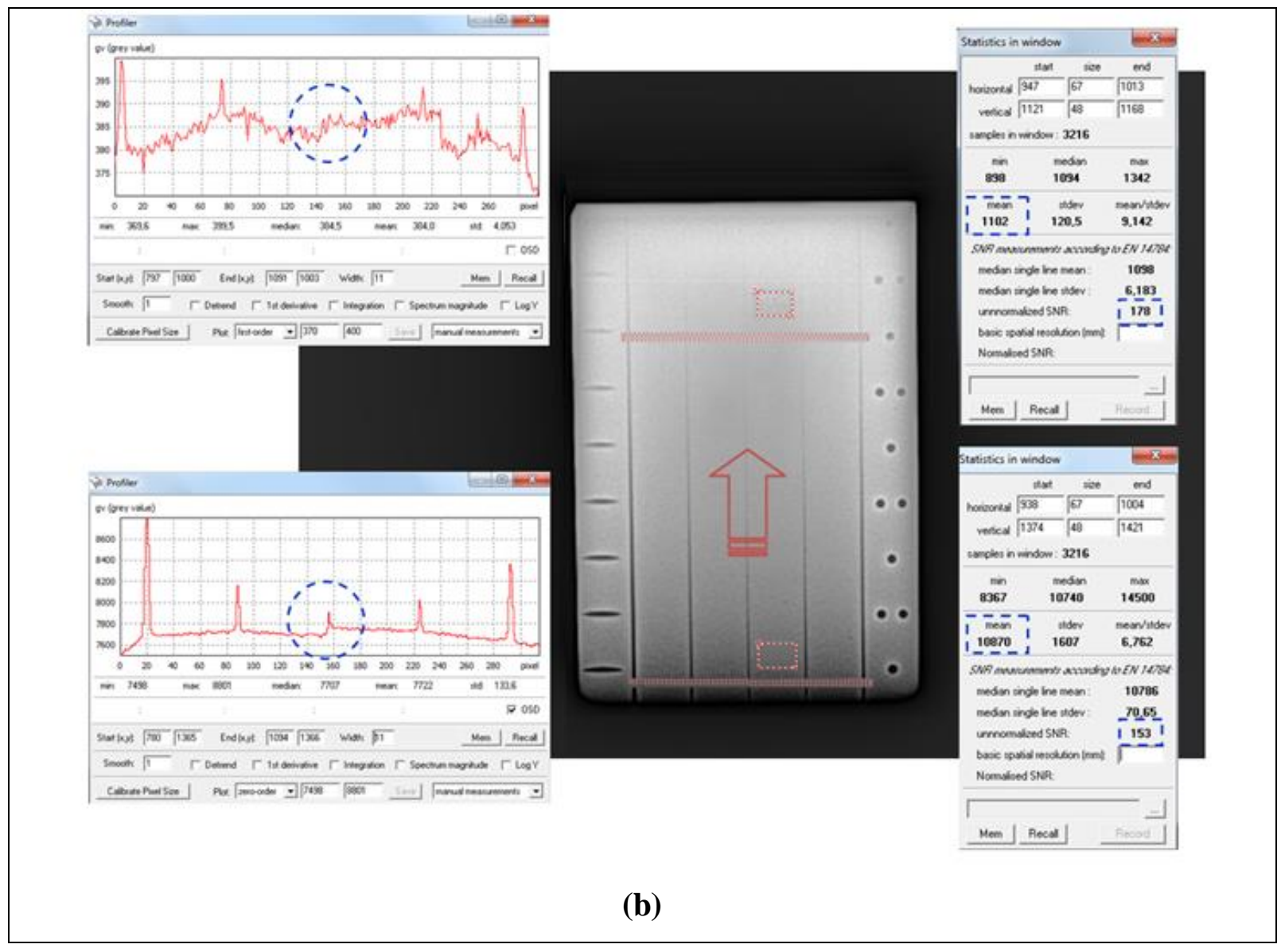

Figure 3: Measurement of quality parameters using: (a) the duplex plate phantom with separate IQIs and (b) the five-groove wedge phantom.

Regarding the pixel map, the DXR250P has a total bad pixel value, including clusters and line segments, almost twelve times higher than the DXR250V. Figure 4 shows the pixel maps to both DDAs systems. The total bad pixel value indicates all pixels identified with a performance outside of the specification range for a pixel of a DDA as defined in Standard ASTM E2597 [11]. The pixel map shows only the dead pixels, that is, pixels that have no response, or that give a constant response independent of radiation dose. There is no standard for acceptance levels for bad pixels, however an assessment should be made of how the prevalence of these anomalous pixels might impact a specific application. These effects were minimized since the images collected for the tests were corrected for bad pixels. 


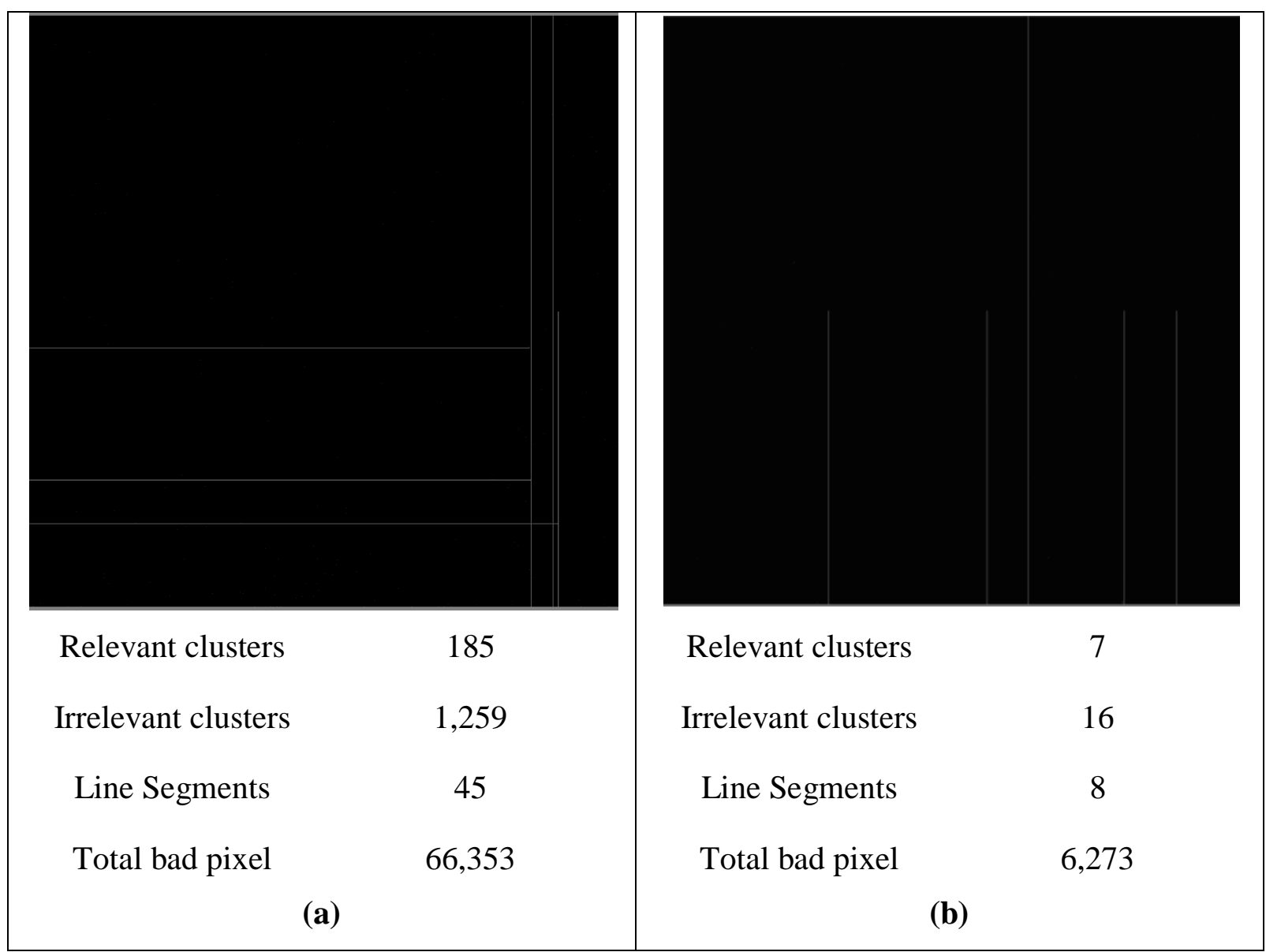

Figure 4: Pixel Map and bad pixel distribution to (a) DXR250P and (b) DXR250V.

Comparing the performance of each DDA system, it is possible to observe that the DXR250P, which have CsI:Tl scintillator, needed a dose almost twice smaller than the DXR250V, which have GOS scintillator, to achieve a similar SL. Furthermore, the DXR250P presented better CS and SNR values. The CsI:Tl scintillator is formed in columnar structures, which restricts the sideways diffusion of optical photons. Moreover, the emission spectrum of CsI:Tl matches well with the absorption response of a-Si [7], achieving a highest light output and, therefore, presenting a higher DQE than GOS screen.

Despite such advantages, it was noted that the DXR250P presents more IL and BI than DXR250V. Figures 5 and 6 show, respectively, the measurements of IL and BI to DDA systems. 


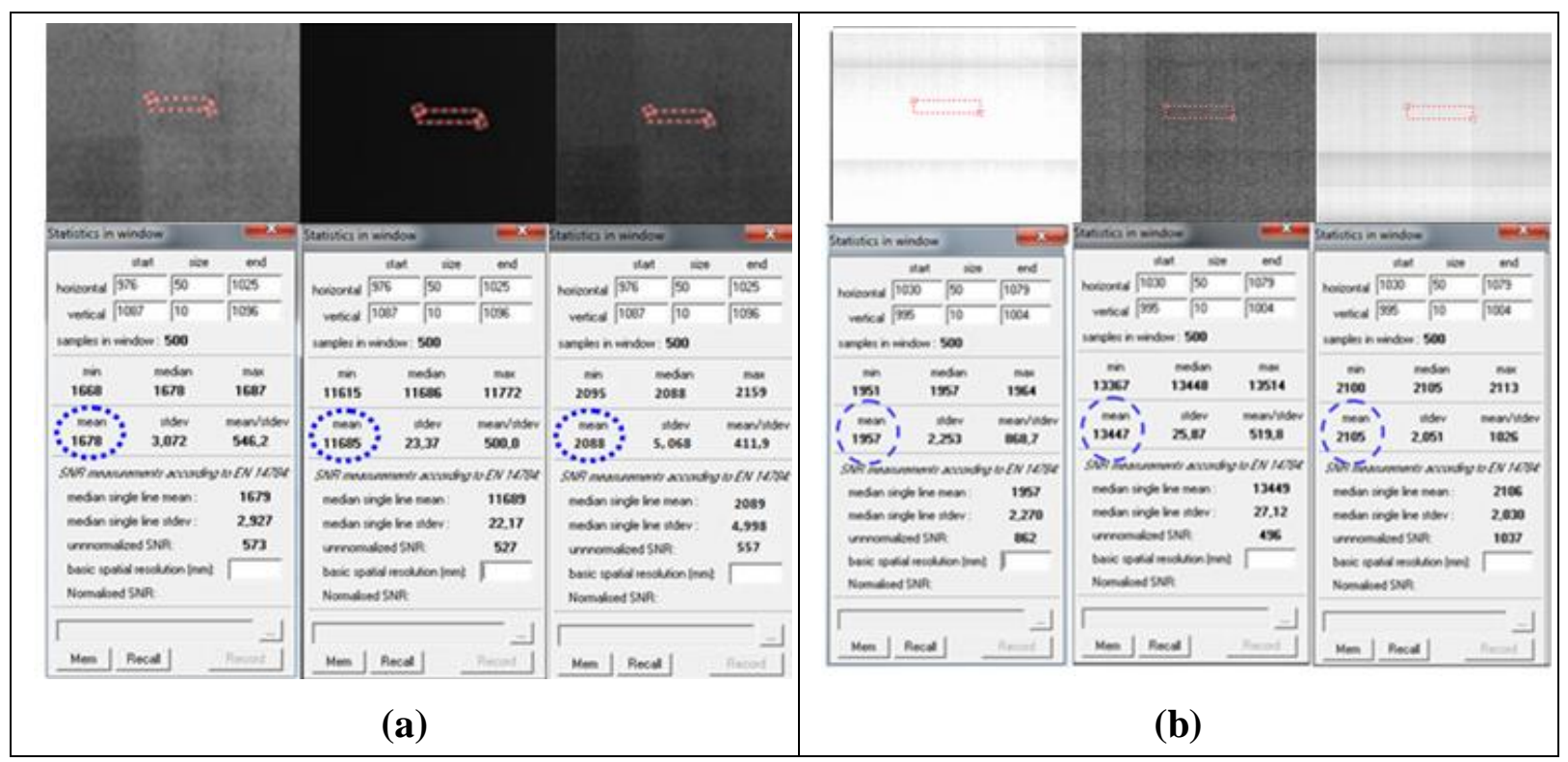

Figure 5: Measurement of IL: (a) DXR250P and (b) DXR250V.

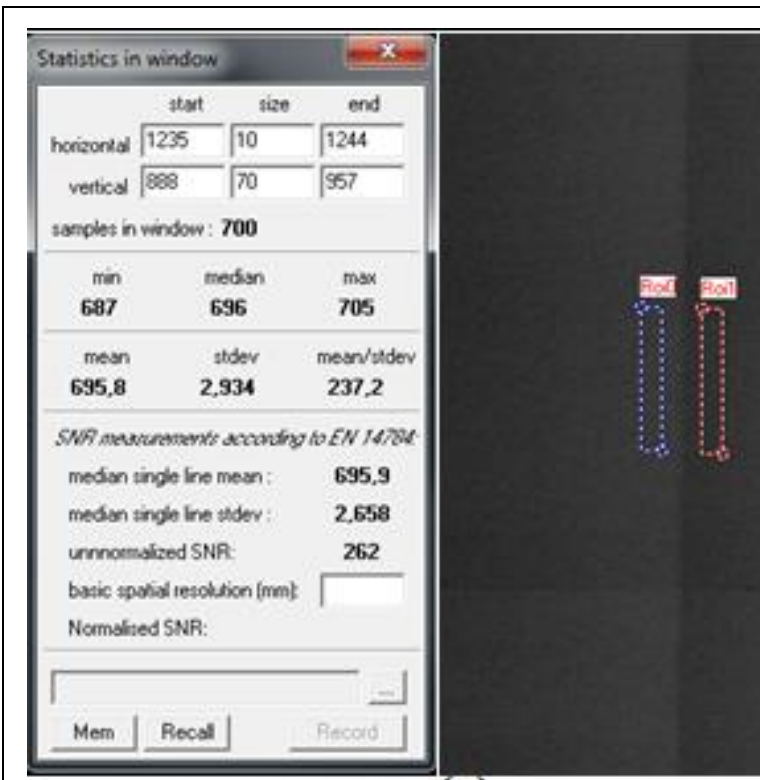

(a)

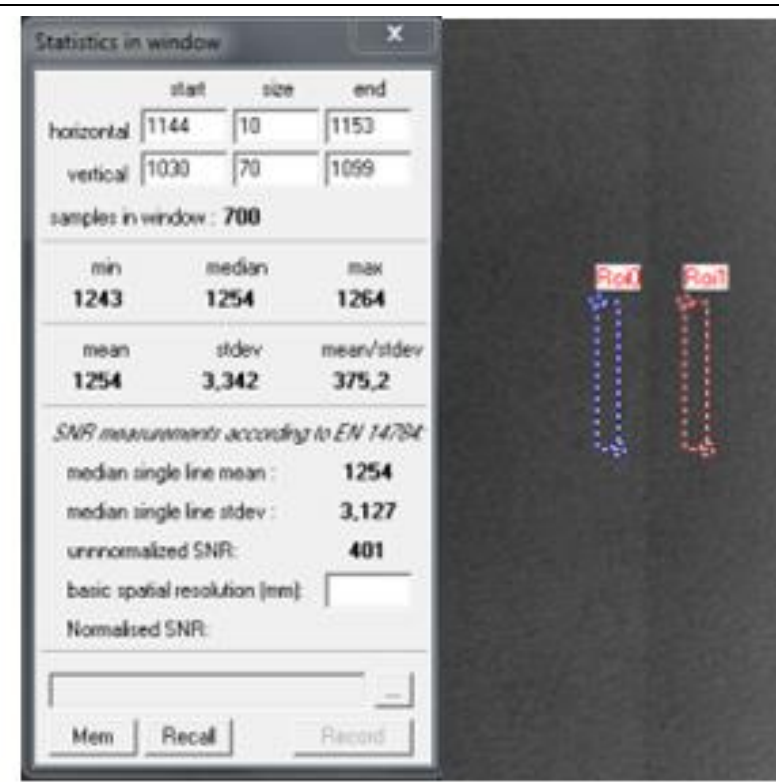

(b)

Figure 6: Measurement of BI: (a) DXR250P and (b) DXR250V.

Lag is a residual signal in the DDA that occurs shortly after the exposure is completed $[8,11]$ and may be affected by several factors, including the type of scintillator. The dominant decay time of the scintillation pulse of CsI:Tl is $680 \mathrm{~ns}$, but its residual signal after the excitation has a characteristic time of $2 \mathrm{~ms}$. Its residual afterglow can be as high as $5 \%$ of the peak value, depending on the intensity and duration of the excitation pulse. At high counting rates, there will be a pulse overlap $[13,14]$. 
Another disadvantage of CsI:Tl is the presence of a hysteresis effect that causes nondeterministic change in its scintillation yield after exposure to high radiation doses. This effect is closely related to Burn In, which is a change in gain of the scintillator that persists well beyond the exposure $[8,11]$. The hysteresis effect can either increase or decrease the light yield during use, representing a substantial source of instability and hence noise [13].

Figure 7 shows the net summary plots of some the measured parameters to the DXR250V and DXR250P to both phantoms. The CS, SL and SNR values presented are for the MTR thickness. These plots provided the knowledge of the quality characteristics of each DDA system analyzed and assist in selecting the type of detector to be used for a given application.

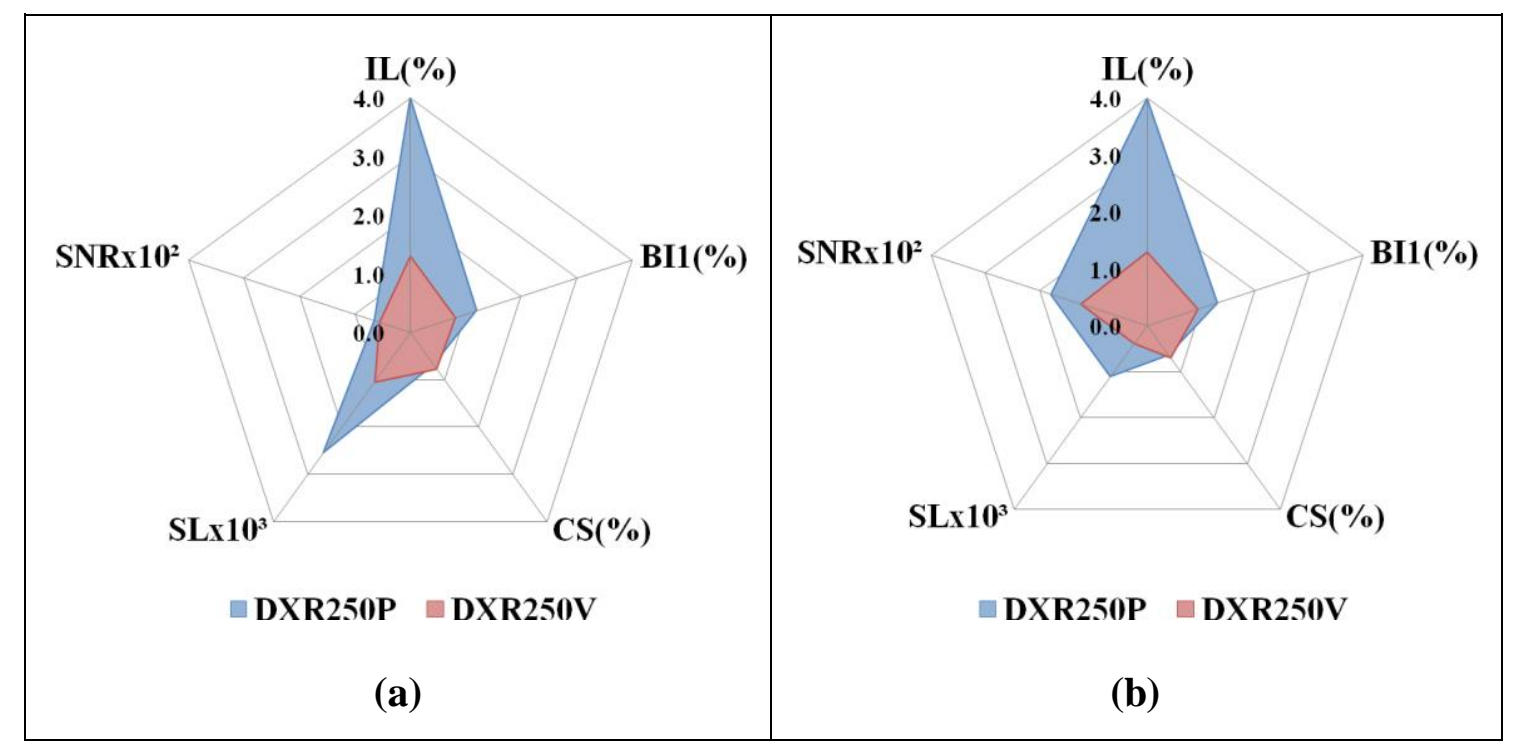

Figure 7: Net summary plots of some the measured parameters: (a) the duplex plate phantom with separate IQIs and (b) the five-groove wedge phantom.

\section{CONCLUSIONS}

The techniques for evaluating the performance of DDA systems used in this work provided satisfactory results for the tests established in the standard ASTM E2737-10. This practice allows both techniques to be used, however it was possible to observe that the use of the fivegroove wedge phantom made the measurements to become easier to execute than the duplex plate phantom with separate IQIs.

The intent of tests presented is to monitor the system performance for degradation and to identify when an action needs to be taken when the system degrades by a certain level. For this reason, independent of the technique performed, it is important that it be maintained for later comparison of the results and verification of long-term system performance.

Regarding the performance of each DDA system, it is possible to observe that the DXR250P, which have CsI:Tl scintillator, is more sensitive, requiring a lower dose to achieve a similar SL than the DXR250V, which have GOS scintillator. Furthermore, the DXR250P presented better CS and SNR, by making this system to generate images with better quality in detail viewing. 
The CsI:Tl has a higher DQE, producing more light output than GOS screen, which results in a higher signal output in the photodiode for a given dose. However, the CsI:Tl scintillator caused the images obtained using the DXR250P to present more IL and BI, which damages the image. The DDA calibration used to generate corrected images is very important to reduce the remaining Lag and Burn In effect.

The results achieved in this paper provided a good understanding of the behavior of each DDA system studied and its influence on the quality of the digital radiographic image. None of the scintillators possesses all the ideal characteristics; both have advantages and limitations in their use. Therefore, the choice of a certain scintillator in a DDA depends strongly on the application and research purpose.

\section{ACKNOWLEDGMENTS}

This work was partially supported by Conselho Nacional de Desenvolvimento Científico e Tecnológico (CNPq), Fundação de Amparo à Pesquisa do Estado do Rio de Janeiro (FAPERJ) and Coordenação de Aperfeiçoamento de Pessoal de Nível Superior (CAPES).

\section{REFERENCES}

1. U. Ewert, U. Zscherpel and K. Bavendiek, "Strategies for Film Replacement in Radiography - Films and Digital Detectors in Comparison", Proceedings of 17th World Conference on Nondestructive Testing, Shanghai, China, 25-28 Oct 2008 (2008).

2. A. R. Cowen, S. M. Kengyelics and A. G. Davies, "Solid-state, flat-panel, digital radiography detectors and their physical imaging characteristics", Clinical Radiology, Vol. 63, pp. 487-498 (2008).

3. W. den Boer, Active Matrix Liquid Crystal Displays, Newnes, Oxford, U.K. (2005).

4. D. F. Oliveira, E. V. Moreira, A. S. S. Silva, J. M. Rebello, R. T. Lopes, M. S. Pereira and U. Zscherpel, "Application of the Digital Radiography in Weld Inspection of Gas and Oil Pipelines", Proceedings of 10th European Conference on Non-Destructive Testing ECNDT, Moscow, Russia, 7-11 Jun 2010 (2010).

5. H. J. Kim, H. K. Kim and G. Cho, J. Choi, "Construction and characterization of an amorphous silicon flat-panel detector based on ion-shower", Nuclear Instruments and Methods in Physics Research A, Vol. 505, pp. 155-158 (2003).

6. R. V. Acharya, U. Kumar and G. Singh, "Experiences in Digital Industrial Radiography using High-Resolution A-Si Flat Panel X-Ray Detector System", Proceedings of the National Seminar \& Exhibition on Non-Destructive Evaluation NDE 2009, Mumbai, India, 10-12 Dec 2009 (2009).

7. H. K. Kim, I. A. Cunningham, Z. Yin and G. Cho, "On the Development of Digital Radiography Detectors: A Review", International Journal of Precision Engineering and Manufacturing, Vol. 9, No. 4, pp. 86-100 (2008).

8. ASTM E 2737, Standard Practice for Digital Detector Array Performance Evaluation and Long-Term Stability (2010).

9. ASTM E 2002, Standard Practice for Determining Total Image Unsharpness in Radiology (1998).

10. ASTM E 1025, Standard Practice for Design, Manufacture, and Material Grouping Classification of Hole-Type Image Quality Indicators (IQI) Used for Radiology (1998). 
11. ASTM E 2597, Standard Practice for Manufacturing Characterization of Digital Detector Arrays (2008).

12. BAM, User Manual for the Measuring Program ISee!,Version 10.2. Available in: http://www.dir.bam.de/ic/ (2017).

13. V. V. Nagarkar, S. C. Thacker, V. Gaysinskiy, L. E. Ovechkina, S. R. Miller, S. Cool and C. Brecher, "Suppression of Afterglow in Microcolumnar CsI:Tl by Codoping With $\mathrm{Sm}^{2+}$ : Recent Advances", IEEE Trans. Nucl. Sci., Vol. 56, No. 3, pp. 565-570 (2009).

14. J. H. Siewerdsen and D. A. Jaffray, "A ghost story: Spatio-temporal response characteristics of an indirect-detection flat panel imager", Medical Physics, Vol. 26, No. 8, pp. 1624-1641 (1999). 